Accelerator Division

Alternating Gradient Synchrotron Department

BROOKHAVEN NATIONAL LABORATORY

Upton, New York 11973

Accelerator Division

Technical Note

AGS/AD/Tech. Note No. 423

\title{
DETERMINING THE CENTER OF THE AGS HIGH FIELD SEXTUPOLE STRING
}

H. Calvani

January 12, 1996 


\title{
Determining the Center of the AGS High Field Sextupole String
}

\author{
Humberto Calvani
}

January 12, 1996

\section{INTRODUCTION}

The purpose of this paper is to further quantify the results for the measurement of the AGS high field sextupole center as presented in AGS Studies Report No. 332 [1]. The sextupole center is defined as the "radius" (i.e. circumference / $2 \pi$ ) or beam frequency at which the betatron tune of the AGS is not influenced by changes in the current in the sextupole string arrays. In the aforementioned study note, both vertical and horizontal betatron tune data were obtained using the Tune Meter program for different values of the $\mathrm{RF}$ frequency on the AGS injection porch. A radial scan was performed for four different horizontal chromaticity settings* $(+1.0,-0.5,-4.0,-2.6)$ obtained by varying the current in the sextupole arrays. Ideally, one would expect the four tune-frequency relationships obtained from the four chromaticity scans and analyzed using the LeastSquares fitting technique to intersect at one point that is then understood as the radius corresponding to the sextupole center. However, because of different factors such as measurement errors in the data, random fluctuations, and insufficient data, this single crossing point was not well-defined. This paper will explore how much deviation in the tune-frequency equations is necessary for these to intersect at one defined point with the constraints of the experimental errors in the data. Further, it will also investigate how the associated Chi-Square $\left(\chi^{2}\right)$ statistic is affected for each individual tune-frequency equation after imposing the constraint of a one point global fit. Such exercise will then yield a specific value for the sextupole center, as well as information about the dispersion of the data with respect to a experimentally constructed "ideal solution" for the AGS high field sextupole center.

\section{Procedure and Results}

The tune-frequency linear equations obtained in the previous work have been re-fitted taking into account errors in both the tune and frequency. The resulting fitted equations along with their associated standard deviations in slope and intercept, $\chi^{2}$ values (measuring the agreement between data and model), and $\chi^{2}$ probabilities (quantitative measure for the goodness of fit of the model) are :

* The study did not treat the two sextupole strings (H\&V) independently. 
Horizontal Tune

$Q_{X}=(21.201 \pm 0.801) f_{R F}-(49.439 \pm 2.818)$

$+1.0$

$\mathrm{Q}_{\mathrm{X}}=(-35.457 \pm 0.335) \mathrm{f}_{\mathrm{RF}}+(106.373 \pm 0.920)$

$Q_{x}=(-6.009 \pm 0.270) f_{R F}+(25.405 \pm 0.745)$

$Q_{X}=(-48.245 \pm 0.584) f_{R F}+(141.547 \pm 1.670)$

\section{Vertical Tune}

$\mathrm{Q}_{\mathrm{y}}=(-38.536 \pm 1.041) \mathrm{f}_{\mathrm{RF}}+(114.855 \pm 2.818)+1.0$

$\mathrm{Q}_{\mathrm{y}}=(-19.064 \pm 2.515) \mathrm{f}_{\mathrm{RF}}+(61.317 \pm 0.691) \quad-4.0$

$\mathrm{Q}_{\mathrm{y}}=(-24.837 \pm 0.328) \mathrm{f}_{\mathrm{RF}}+(77.189 \pm 0.902) \quad-0.5$

$\mathrm{Q}_{\mathrm{y}}=(-8.532 \pm 0.336) \mathrm{f}_{\mathrm{RF}}+(32.349 \pm 0.924) \quad-2.6$ $\chi 2$

53.5

16.5

970.9

42.2

32.7

185.5

322.0

21.8 $\chi 2$ Prob

$8.6 \times 10^{-9}$

$3.6 \times 10^{-2}$

0.0

$9.8 \times 10^{-7}$

Here $f_{R F}$ denotes the RF frequency measured in $\mathrm{MHz}$. It is important to note the magnitude of the $\chi^{2}$ and $\chi^{2}$ probability values obtained for the fits. In general, for a fairly good fit a typical value of $\chi^{2} \approx v$, where $v=N-M$, represents the number of degrees of freedom*[2]. For the data under study we would have expected $\chi^{2} \approx 8$ for each fit. Small $\chi^{2}$ probabilities values $\left(\chi^{2}<10^{-3}\right)$, on the other hand, are generally understood as indication of either a statistically wrong model or an underestimation in measurement errors. For our results neither is the case. Apart from some small deviations from a linear model (except for $\xi=-0.5$ which are large), the resulting large Chi-Square, and thus small Chi-Square probabilities values, can be attributed to the fact that in the process of fitting a straight line model $\mathrm{y}(\mathrm{x})=\mathrm{ax}+\mathrm{b}$ with standard deviations $\sigma_{\mathrm{X}}$ and $\sigma_{\mathrm{y}}$, the Chi-Square function takes the form [2]

$$
\chi^{2}(a, b)=\sum_{i}\left(y_{i}-a x_{i}-b\right)^{2} /\left(\sigma_{y i}^{2}+a^{2} \sigma_{x i}^{2}\right)
$$

As can be seen, the Chi-Square function scales inversely proportional to the squares of the errors. For the data in question, the error in the betatron tune was arbitrarily set to $10^{-3}$, which is the limit of accuracy posed by the Tune Meter program if the produced fast Fourier transform of the kicked beam yielded a well defined sharp peak with a delta function like-nature [3]. In reality the problem is more complicated since we are attempting to determine the mean of a non-uniform spectral distribution of possible betatron tunes**. Thus, it is important to keep in mind that the betraton tune uncertainty could lie above or below this limit.

The Frequency Meter, on the other hand, limited the accuracy of the frequency measurements to $\sim 10^{-6} \mathrm{MHz}$. Therefore, in the process of least square fitting eq.(1) with errors of such accuracy one finds that the value of $\chi^{2}$ is overestimated, and therefore its value should be taken with caution. Inspection of the plots (Figures 1 and 2), reveals that the data is reasonably well correlated with a linear model. As such, we

\footnotetext{
${ }^{*} \mathrm{~N}=$ \# of data points, i.e. for the data in question, $\mathrm{N}=10$; and $\mathrm{M}=$ \# of parameters to be obtained in the fitting process, i.e. 2 , slope and intercept.

** This is a limitation present in the Tune Meter program. It would be of great importance to modified the code to yield an estimate of the significance (probability) of the maximum peak against the hypothesis of random noise.
} 
cannot rely on the Chi-Square statistic as an "absolute" measure of how well the data fits the model. Rather, we will make use of it when comparing the results of the global fit, thereby measuring the amount of relative depreciation or improvement in $\chi^{2}$ that was required in order to obtain the intersection.

Figures 1 and 2 show the data and the best fitted line for the horizontal and vertical betatron tunes respectively. Error bars were not displayed since these would not be observable in the corresponding scale. As one can conclude from the figures, there is not a single well defined crossing point corresponding to the sextupole center in either plane. In order to require the tune-frequency equations to intersect at a single point, the following algorithm is constructed. Given a possible crossing point $\left(\mathrm{x}_{0}, \mathrm{y}_{0}\right)$, we can use the point-slope equation of a line $a\left(x_{i}-x_{0}\right)-\left(y_{i}-y_{0}\right)=0$, to find the best tunefrequency equation (for each chromaticity setting) to allow for the global intersection. This operation is to be performed simultaneously for the four equations in each betatron tune plane. Thus, one can design the Chi-Square global function $\chi_{\mathrm{G}}{ }^{2}$

$$
\begin{aligned}
\chi_{\mathrm{G}}^{2}{ }_{\left(\mathrm{a}_{1}, \mathrm{a}_{2}, \mathrm{a}_{3}, \mathrm{a}_{4}, \mathrm{x}_{0}, \mathrm{y}_{0}\right)=} & \sum_{\mathrm{i}, \xi=+1.0}\left[\mathrm{a}_{1}\left(\mathrm{x}_{\mathrm{i}}-\mathrm{x}_{0}\right)-\left(\mathrm{y}_{\mathrm{i}}-\mathrm{y}_{0}\right)\right]^{2} /\left(\sigma_{\mathrm{yi}}{ }^{2}+\mathrm{a}_{1}{ }^{2} \sigma_{\mathrm{xi}}{ }^{2}\right)+ \\
& \sum_{\mathrm{i}, \xi=-4.0}\left[\mathrm{a}_{2}\left(\mathrm{x}_{\mathrm{i}}-\mathrm{x}_{0}\right)-\left(\mathrm{y}_{\mathrm{i}}-\mathrm{y}_{0}\right)\right]^{2} /\left(\sigma_{\mathrm{yi}}{ }^{2}+\mathrm{a}_{2}{ }^{2} \sigma_{\mathrm{xi}}{ }^{2}\right)+ \\
& \sum_{\mathrm{i}, \xi=-0.5}\left[\mathrm{a}_{3}\left(\mathrm{x}_{\mathrm{i}}-\mathrm{x}_{0}\right)-\left(\mathrm{y}_{\mathrm{i}}-\mathrm{y}_{0}\right)\right]^{2} /\left(\sigma_{\mathrm{yi}}{ }^{2}+\mathrm{a}_{3}{ }^{2} \sigma_{\mathrm{xi}}{ }^{2}\right)+ \\
& \sum_{\mathrm{i}, \xi=-2.6}\left[\mathrm{a}_{4}\left(\mathrm{x}_{\mathrm{i}}-\mathrm{x}_{0}\right)-\left(\mathrm{y}_{\mathrm{i}}-\mathrm{y}_{0}\right)\right]^{2 /\left(\sigma_{\mathrm{yi}}{ }^{2}+\mathrm{a}_{4}{ }^{2} \sigma_{\mathrm{xi}}{ }^{2}\right),}
\end{aligned}
$$

describing the sum of squares for each point slope equation [4], where, the $a_{i}$ correspond to the slope of each global fitted tune-frequency equation, and the $x_{i}$ and $y_{i}$ the RF frequency and betatron tune of the $i$ th data point respectively.

We can then utilize the least squares method and minimize the latter expression in order to obtain the values for the slopes $\left(\mathrm{a}_{\mathrm{i}}\right)$. Consequently, one can refer back to the point-slope equation of a line, which then coupled with knowledge of the crossing point $\left(\mathrm{x}_{0}, \mathrm{y}_{0}\right)$, attains the equation of the line which yields the global fit.

The best crossing point $\left(\mathrm{x}_{0}, \mathrm{y}_{0}\right)$ is the one that, combined with the values for the $a_{i}$, minimizes eq. (2) above. The search for this point requires the construction of a data grid in the region of interest, which will give eq.(2) the freedom to search for the best minimum in tune-frequency space. Figures 3 and 4 display the $20 \times 20$ data grids which were used in this search. For both the horizontal and vertical betatron tune cases, the tune was allowed to range from $8.80 \leq \mathrm{Q}_{\mathrm{x}, \mathrm{y}} \leq 8.93$ with a resolution of 0.0068 , and for the frequency $2.748 \leq \mathrm{f}_{\mathrm{RF}} \leq 2.752$, in steps of $0.0002 \mathrm{MHz}$. From here, we can then proceed to minimize eq. (2) using the steepest descent method, which searches for the minimum (at least a local minimum although not necessarily global) by following the path of steepest descent along the $\chi^{2}$ surface.

Figures 5 and 6 show the resultant global fit superimposed on the best fitted lines. The best crossing point in the horizontal grid corresponded to $\mathrm{Q}_{\mathrm{x}}=8.8748$, $f_{\mathrm{RF}}=2.7500 \mathrm{MHz}$, and in the vertical case to $\mathrm{Q}_{\mathrm{y}}=8.8952$ and $\mathrm{f}_{\mathrm{RF}}=2.7496 \mathrm{MHz}$. Refer to Figures 7 and 8 for a closer examination of the global fit. Additionally, it is 
also of interest to view each individual equation in a separate plot in order to get a pictorial idea of the amount of deviation that was necessary in order to intersect the lines. Figures 9-12 display the fits for each chromaticity setting of the horizontal tune case. Similarly, Figures 13-16 show the results for the vertical tune calculation.

The resulting global-fitted equations with their respective $\chi_{\mathrm{G}}{ }^{2}$ values are displayed below. The $\chi^{2}$ values from the fits to the data and dispersions from the global fit ( $\Delta \chi_{\mathrm{f}}^{2}=\chi_{\mathrm{G}}{ }^{2}-\chi^{2}$ ) are also included for purposes of comparison.

\section{Horizontal Tune}

$\mathrm{Q}_{\mathrm{x}}=14.138 \mathrm{f}_{\mathrm{RF}}-30.005$

$\mathrm{Q}_{\mathrm{X}}=-38.988 \mathrm{f}_{\mathrm{RF}}+116.091$

$\mathrm{Q}_{\mathrm{x}}=-4.994 \mathrm{f}_{\mathrm{RF}}+22.607$

$Q_{x}=-48.819 f_{R F}+143.129$

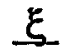

$+1.0$

$-4.0$

$-0.5$

$-2.6$
$\chi_{\underline{G} \underline{2}}$

610.9

317.5

1286.5

44.1 $x 2$

53.5

16.5

970.9

42.2
$\Delta \mathbf{x}_{\underline{f}}$

557.4

301.0

315.6

1.9

\section{Vertical Tune}

$\begin{array}{lllll}Q_{y}=-37.849 f_{R F}+112.967 & +1.0 & 33.4 & 32.7 & 0.7 \\ Q_{y}=-17.636 f_{R F}+57.564 & -4.0 & 265.9 & 185.5 & 80.4 \\ Q_{y}=-24.247 f_{R F}+75.564 & -0.5 & 335.0 & 322.0 & 13.0 \\ Q_{y}=-10.140 f_{R F}+36.776 & -2.6 & 50.6 & 21.8 & 28.8\end{array}$

\section{Discussion and Conclusion}

As can be observed from the above results, constraining the tune equations to intersect at one point did not severely affect the quality of the fits, except for settings $\xi=+1.0, \xi=-4.0$ (Figure 9 and 10 respectively), and $\xi=-0.5$ (Figure 11) in the horizontal tune plane, which reflect considerable subsequent depreciation in $\chi^{2}$.

The large $\Delta \chi_{\mathrm{f}}^{2}$ dispersion for $\xi=+1.0, \xi=-4.0$ is surprising, since the best fitted data is clearly linear but it properties are such that it does not intersect at the same point. This serves to indicate that the model is wrong. The reason for this incompability is not clearly understood and this will be investigated in future work.

For $\xi=-0.5$ : horizontal tune (Figure 11), the obtained large disagreement between the best fit and the global fitted data is however anticipated since the behavior of the data departs largely from a linear model, without a clear explanation of what is responsible for this phenomena. Additional data for this drill, as well as subsequent sets of data would be essential for a clearer interpretation of the observed shape.

Further examination of the results obtained above shows that the global fit yielded values for the slope and intercept in the equations, which, except for $\xi=-2.6$ in horizontal string and $\xi=+1.0$ in vertical string, did not fall within the $(1 \sigma: 68 \%$ confidence level) standard deviations for the fitted equations to the data. This can be attributed to the small error bars in the data, the size of the data grid and the resolution used. Since for each different crossing point $\left(\mathrm{x}_{0}, \mathrm{y}_{0}\right)$, there will be a correspondingly different numerical value for the slope $\left(a_{i}\right)$ and intercept, the size of the data grid and/or the more $\left(\mathrm{x}_{0}, \mathrm{y}_{0}\right)$ points that are sampled ( same data-grid area but increasing 
resolution), the more likely $\chi_{G}{ }^{2}$ would converge to a minimum with values of slope and intercept approaching or deviating from those obtained in the best-fitted equations.

Given the values for the center frequency (corresponding to $R_{0}$, the equilibrium orbit radius) at which the intersection occurs, we can conclude that the center for the horizontal and vertical string array differ by $400 \mathrm{~Hz}(\sim 2 \mathrm{~mm})$. One might question this difference, having expected the centers to be equal for both sextupole strings. However, this is not a requisite. Further, as mentioned above, this difference could be attributed to lack of resolution in the data grid used. Nevertheless, this is a considerable difference, and taking into account the uncertainty in the frequency measurements ( $\sim 10^{-6} \mathrm{MHz}$ ), the resulting standard deviations for the sextupole centers $(\sim 0.05 \mathrm{~mm})$ do not allow the central values to overlap.

In the previous work, the obtained crossing frequency extracted from the horizontal data was $f_{R F}=2.7501 \mathrm{MHz}$, with an uncertainty of $0.1 \mathrm{KHz}$, which then, considering the errors, is in agreement with the results presented here $\left(f_{R F}=2.7500\right.$ MHz).

We are now also able to calculate the relative position ("zero") of the AGS Orbit system. Using the obtained equation from the previous work relating RF frequency with radial average, namely

$$
f_{\mathrm{RF}}=2.752024+0.0037165<\mathrm{R}_{\mathrm{ave}}>\quad[\mathrm{MHz}]
$$

and noting the calibration for the AGS Orbit program (where 1 "cm" radial shift corresponds to $1.86 \mathrm{~cm}$ ), we can determine the zero for the horizontal and vertical string to be $10.138 \mathrm{~mm}$ and $12.131 \mathrm{~mm}$ respectively, and to the of outside of $R_{0}$.

This result differs slightly with the estimate obtained in the studies note for the central value of the horizontal string, where the latter was found to be $9.6 \mathrm{~mm}$. However, as mentioned above, this figure is in agreement when the uncertainties are included. Accordingly, these results stress the existing disagreement with what is believed to correspond to the "Beam Code Axis" for the AGS orbit system ( $\sim 4 \mathrm{~mm}$ outside of $\left.R_{0}\right)$, therefore supporting the speculation raised in the studies note, whereby such inconsistency is to be expected if the radial offsets in the Orbit program have not been corrected to follow the centering of the PUE plates in the vaccum chambers of the AGS.

To improve upon the study as conducted thus far, the following points should be addressed: (i) The horizontal and vertical sextupole strings should be treated independently. (ii) Would the change in the equilibrium orbit due to the change in chromaticity, meet the requirement of the basic assumption of the model definition of the sextupole center? (iii) In the interest to further explore the validity of our model, how significant is the second order effect of a $9^{\text {th }}$ harmonic component (since the AGS betatron tune runs close to a $9^{\text {th }}$ integer) in producing the observed intersection offset in the tune-frequency equations for both sextupole strings? Future work is needed in order to further probe and gain understanding on the nature of this problem.

I thank Leif Ahrens and Mike Blaskiewicz for many stimulating and beneficial discussions regarding this work. 


\section{References}

[1] L. Ahrens and C. Whalen, Measurement of the Sextupole String "Center" on the AGS Injection Porch, AGS Studies Report No.332, July 11, 1995.

[2] Press et al.,Numerical Recipes, Cambridge 1992.

[3] Discussions with Leif Ahrens.

[4] Discussions with Mike Blaskiewicz. 


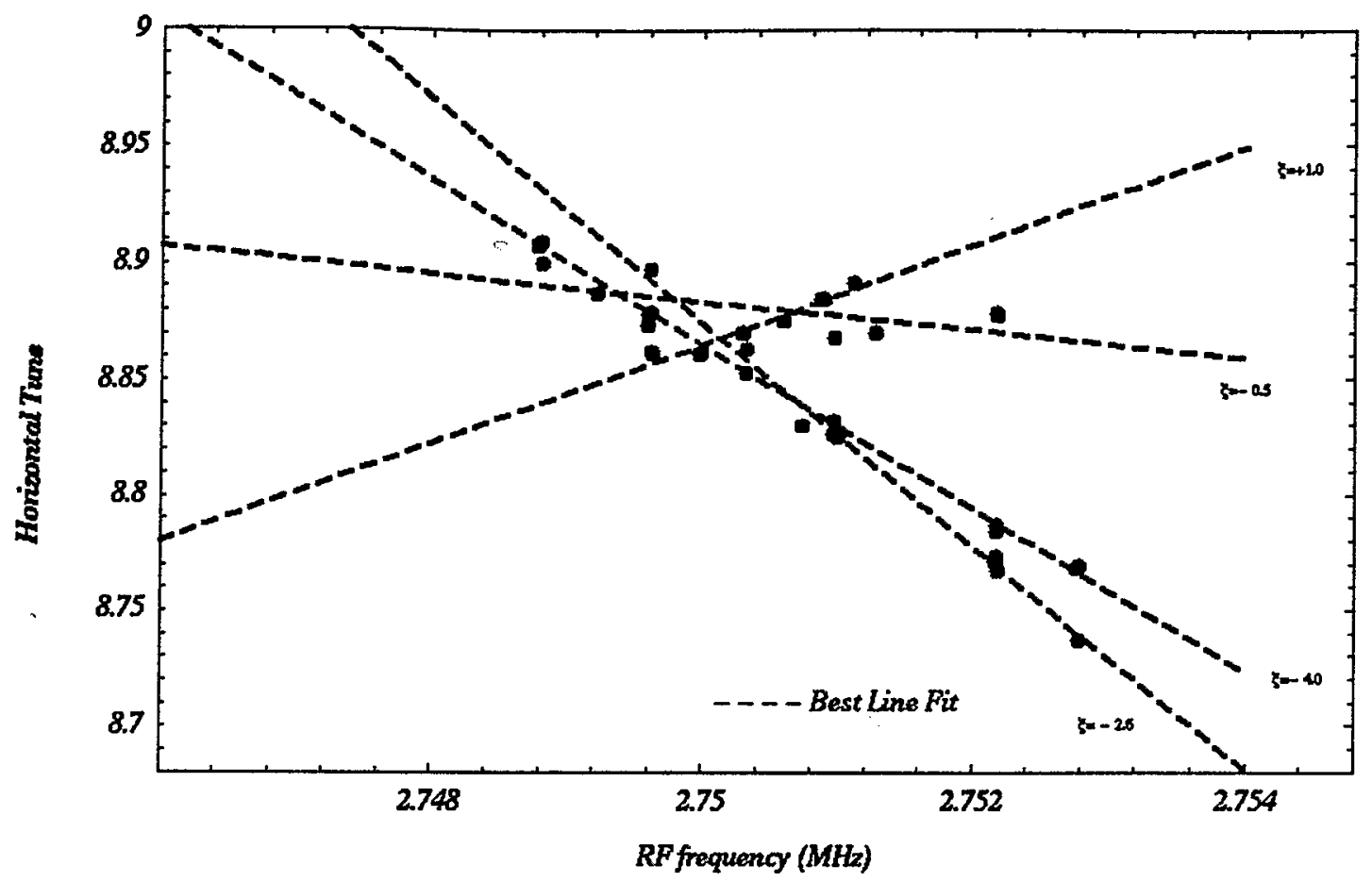

Figure 1 : Horizontal tune data and best fitted equations for the four horizontal chromaticity settings $(+1.0,-0.5,-4.0,-2.6)$.

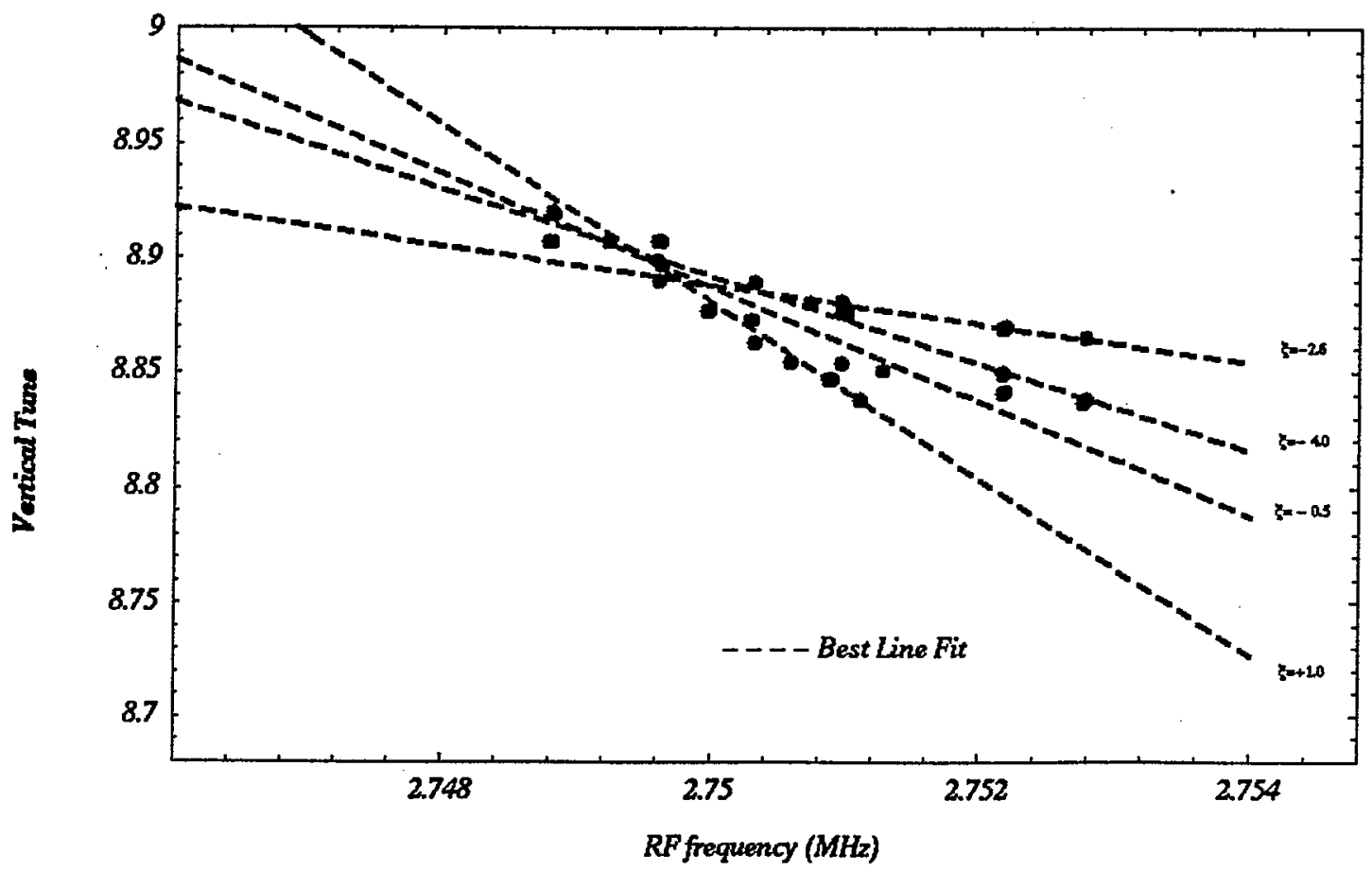

Figure 2 : Vertical tune data and best fitted equations for the four horizontal chromaticity settings $(+1.0,-0.5,-4.0,-2.6)$. 


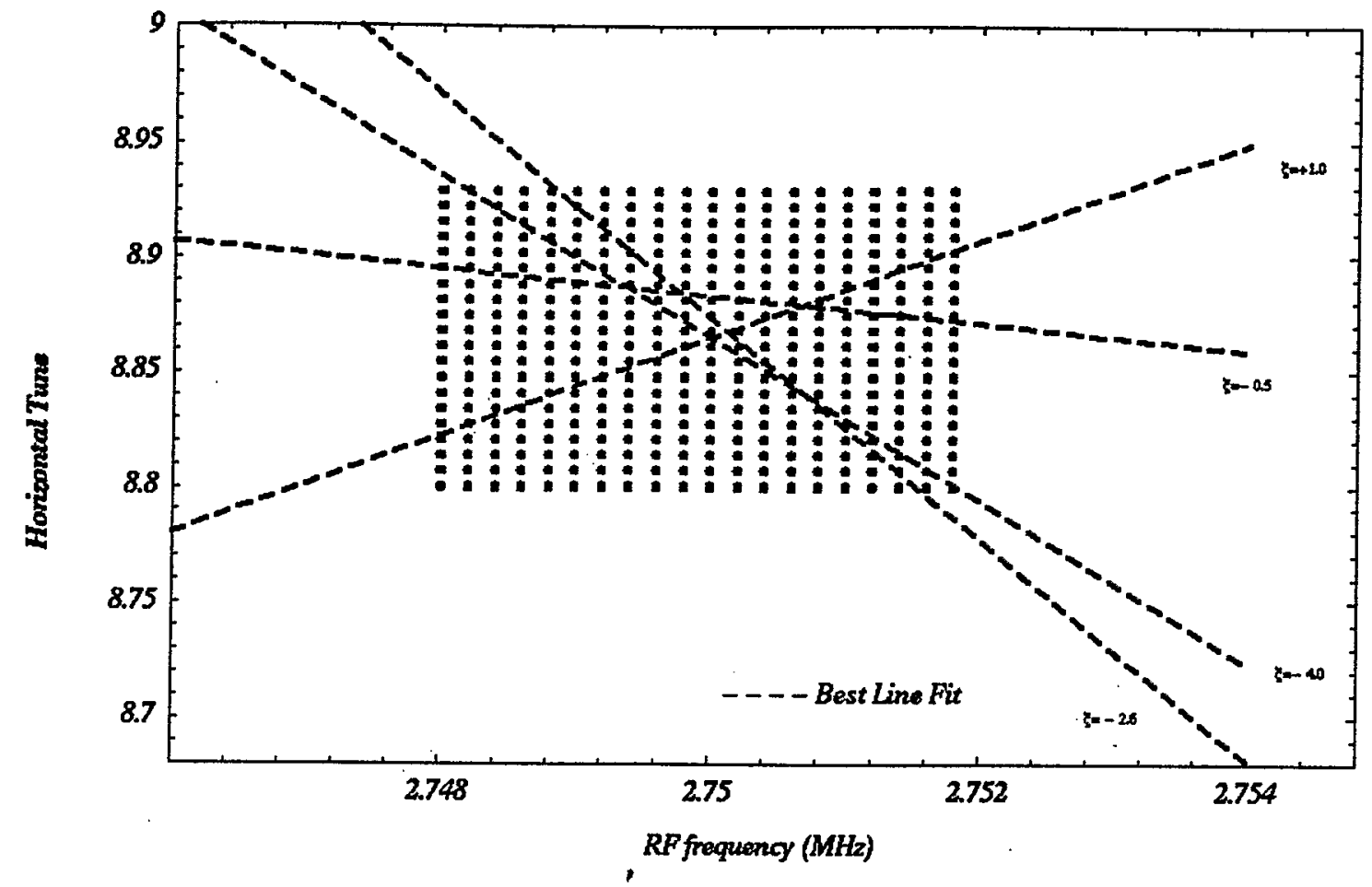

Figure $3: 20 \times 20$ data grid used in search for best crossing point (center) in the horizontal sextupole string.

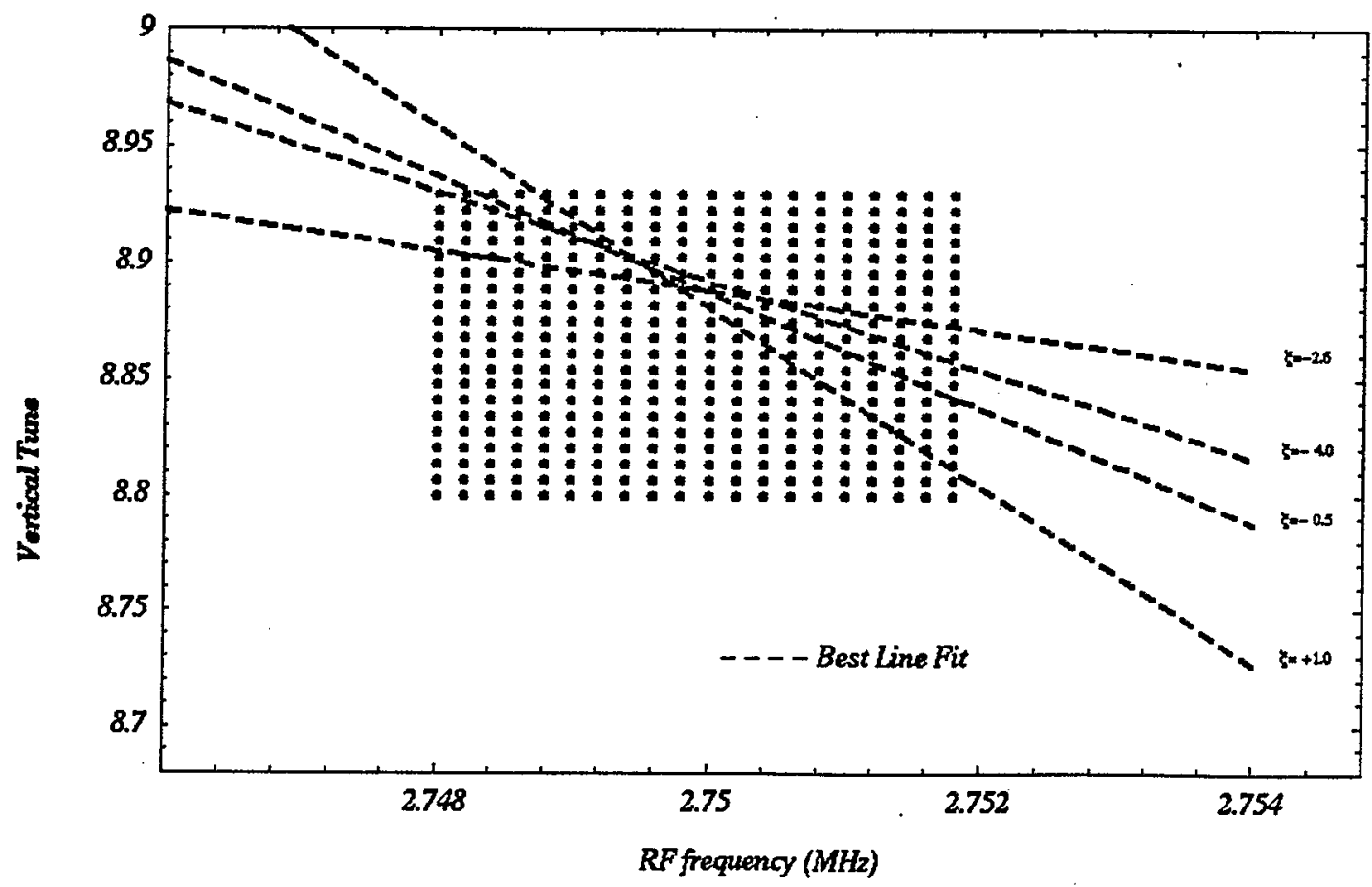

Figure $4: 20 \times 20$ data grid used in search for best crossing point (center) in the vertical sextupole string. 


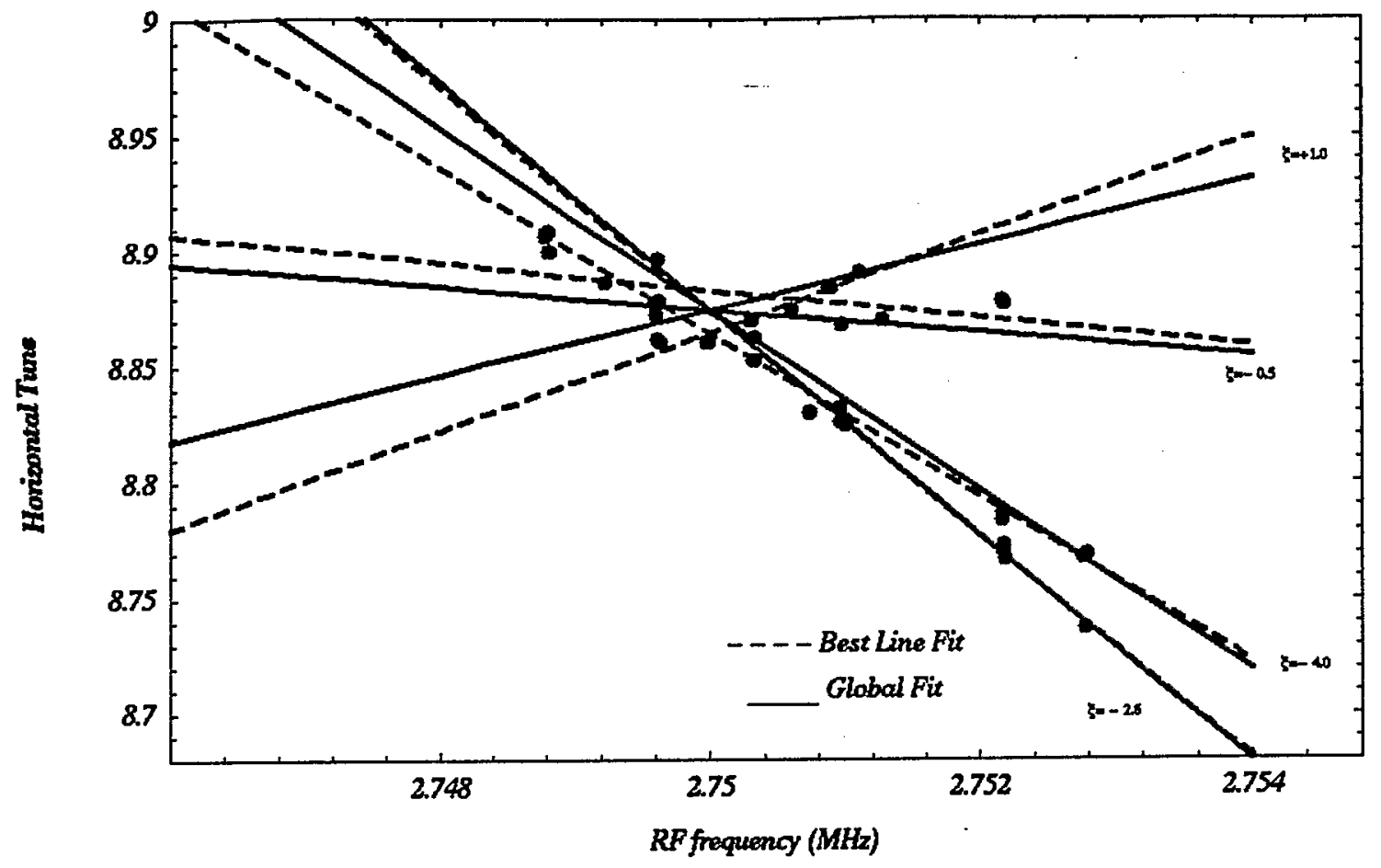

Figure 5 : Global fit for horizontal string displaying the intersection point $\left(f_{R F}, Q_{X}\right)=(2.7500,8.8748)$ resulting from the minimization of eq.(2).

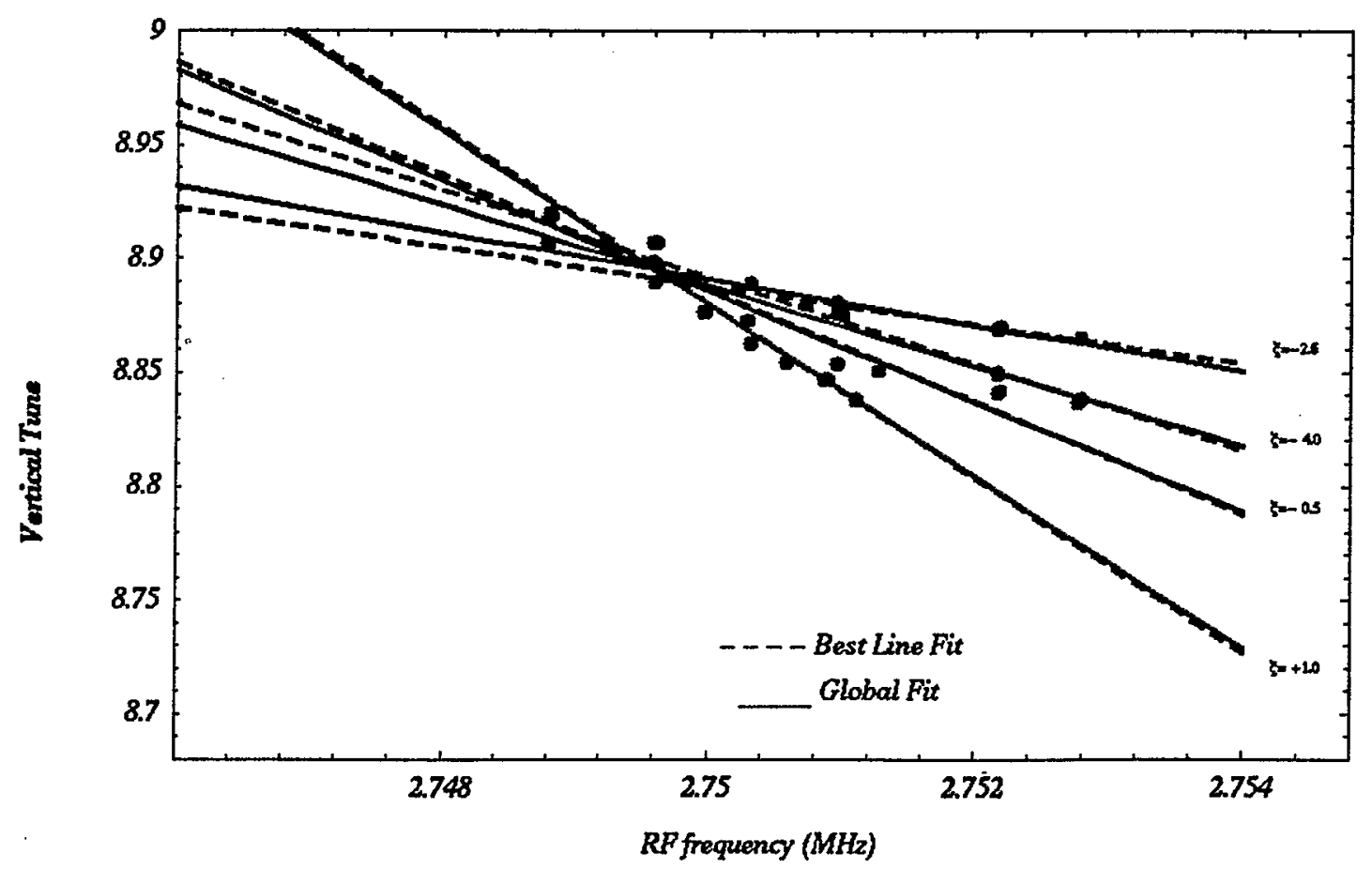

Figure 6 : Global fit for vertical string displaying the intersection point $\left(f_{R F}, Q_{y}\right)=(2.7496,8.8952)$ resulting from the minimization of eq. $(2)$. 

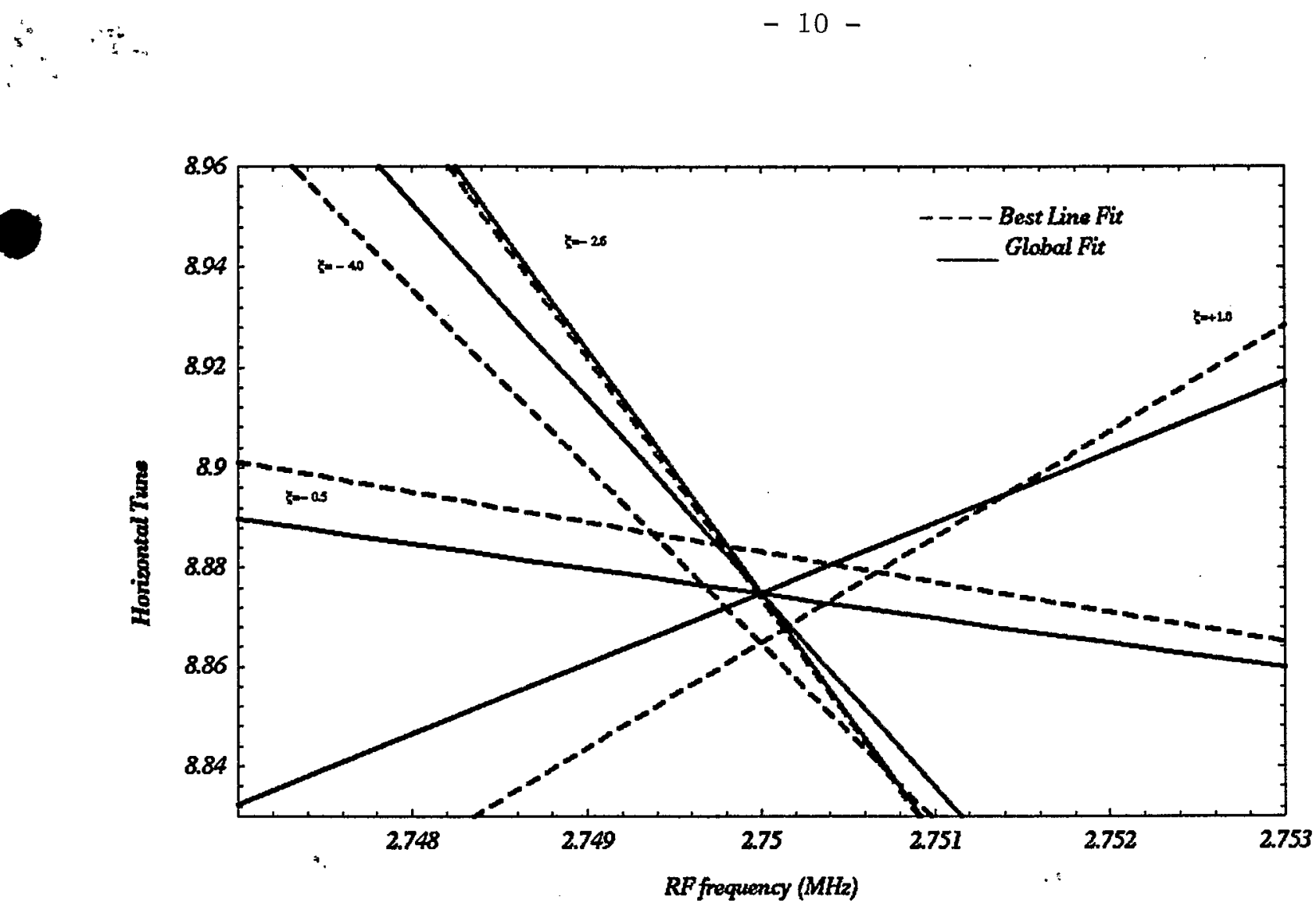

Figure 7: $\quad$ Enlargement of Figure 5

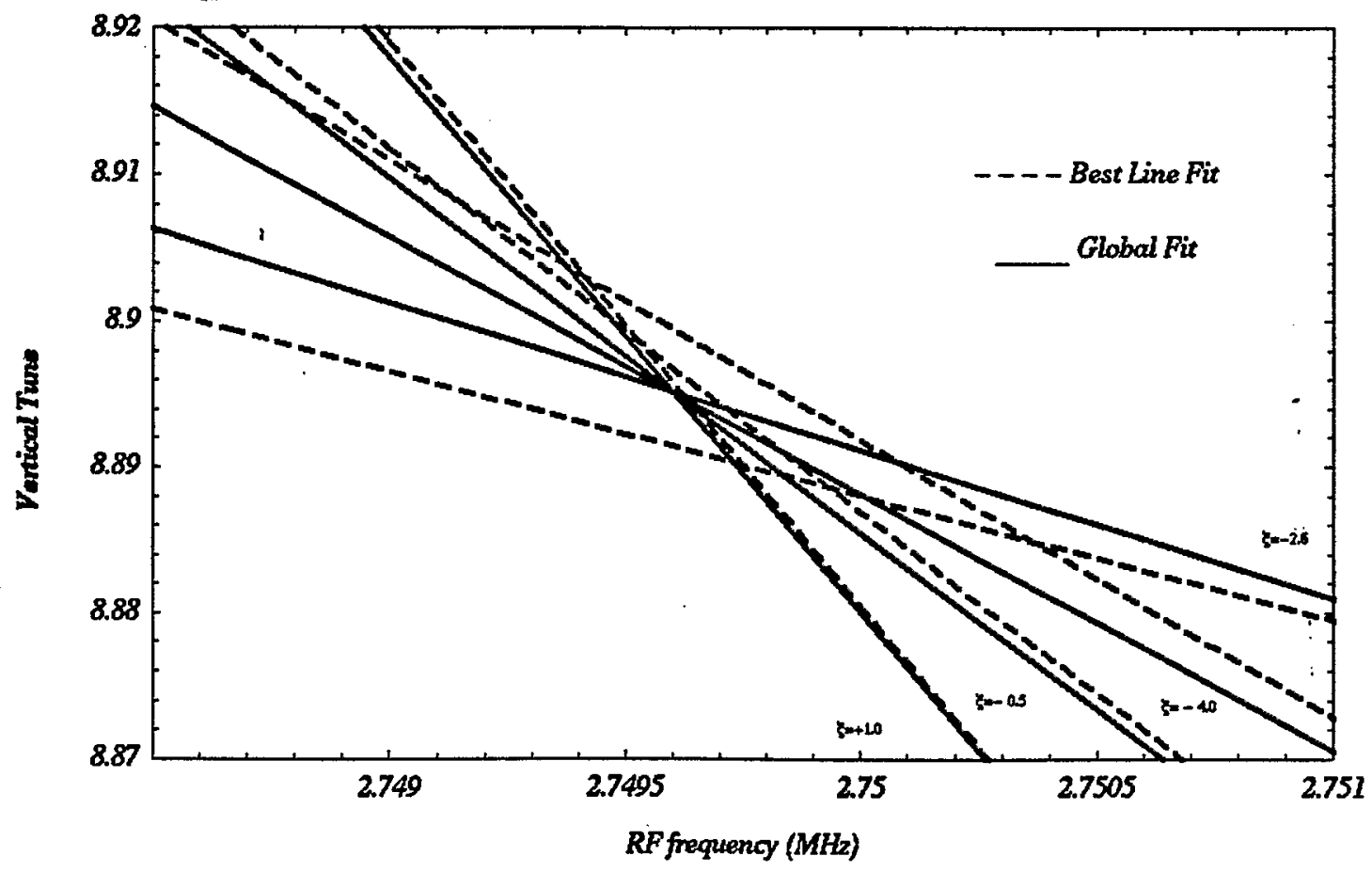

Figure 8 :

Enlargement of Figure 6 
$\pm$

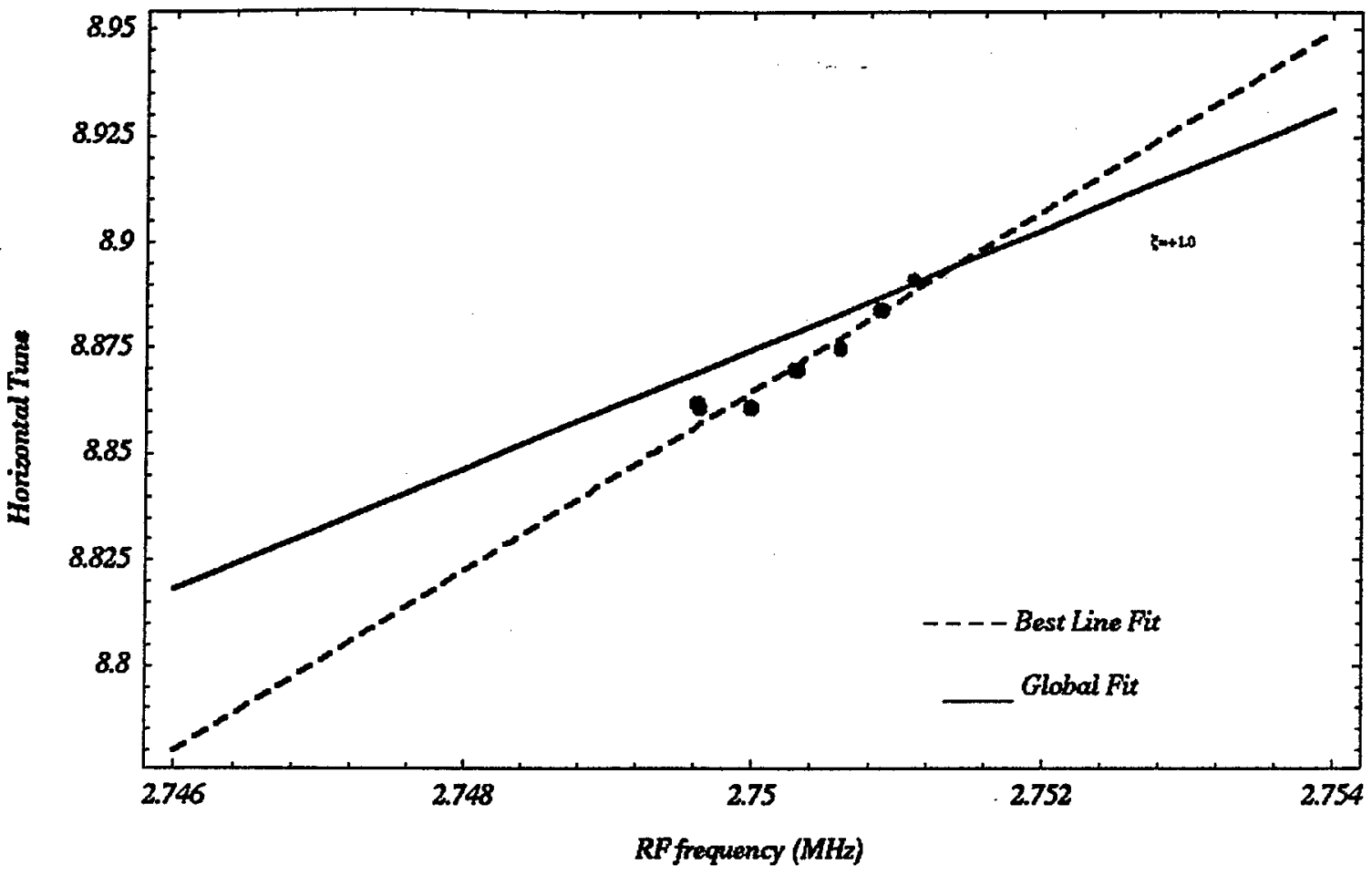

Figure 9 : Individual plot for best-fitted and global fitted equations for horizontal tune and $\xi=+1.0$. The dispersion between global fit and best-fitted equations corresponds to $\Delta \chi_{\mathrm{f}}^{2}=557.4$

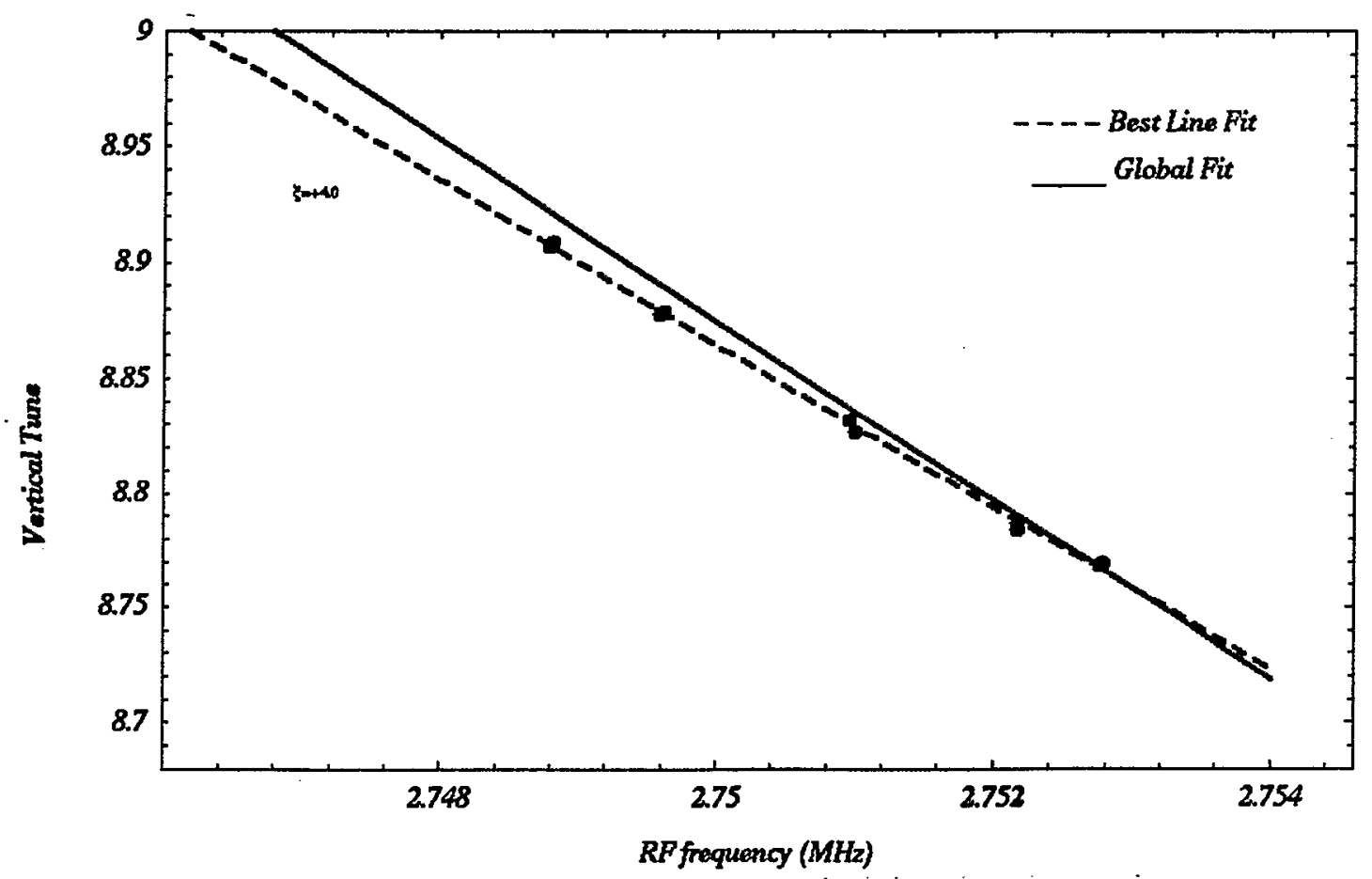

Figure 10 : Individual plot for best-fitted and global fitted equations for horizontal tune and $\xi=-4.0$. The dispersion between global fit and best-fitted equations corresponds to $\Delta \chi_{\mathrm{f}}^{2}=301.0$ 
i.

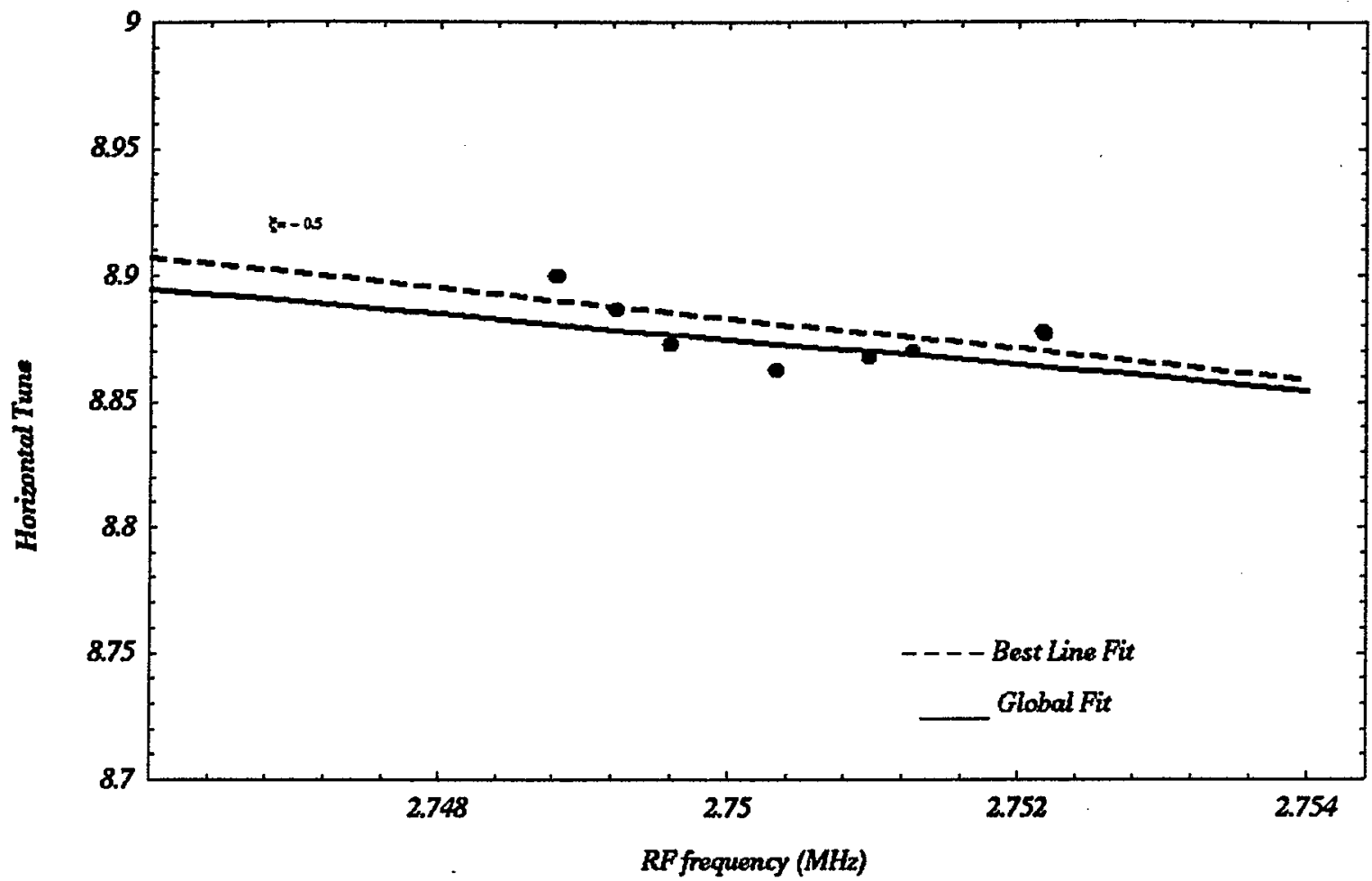

Figure 11 : Individual plot for best-fitted and global fitted equations for horizontal tune and $\xi=-0.5$. The dispersion between global fit and best-fitted equations corresponds to $\Delta \chi_{f}^{2}=315.6$

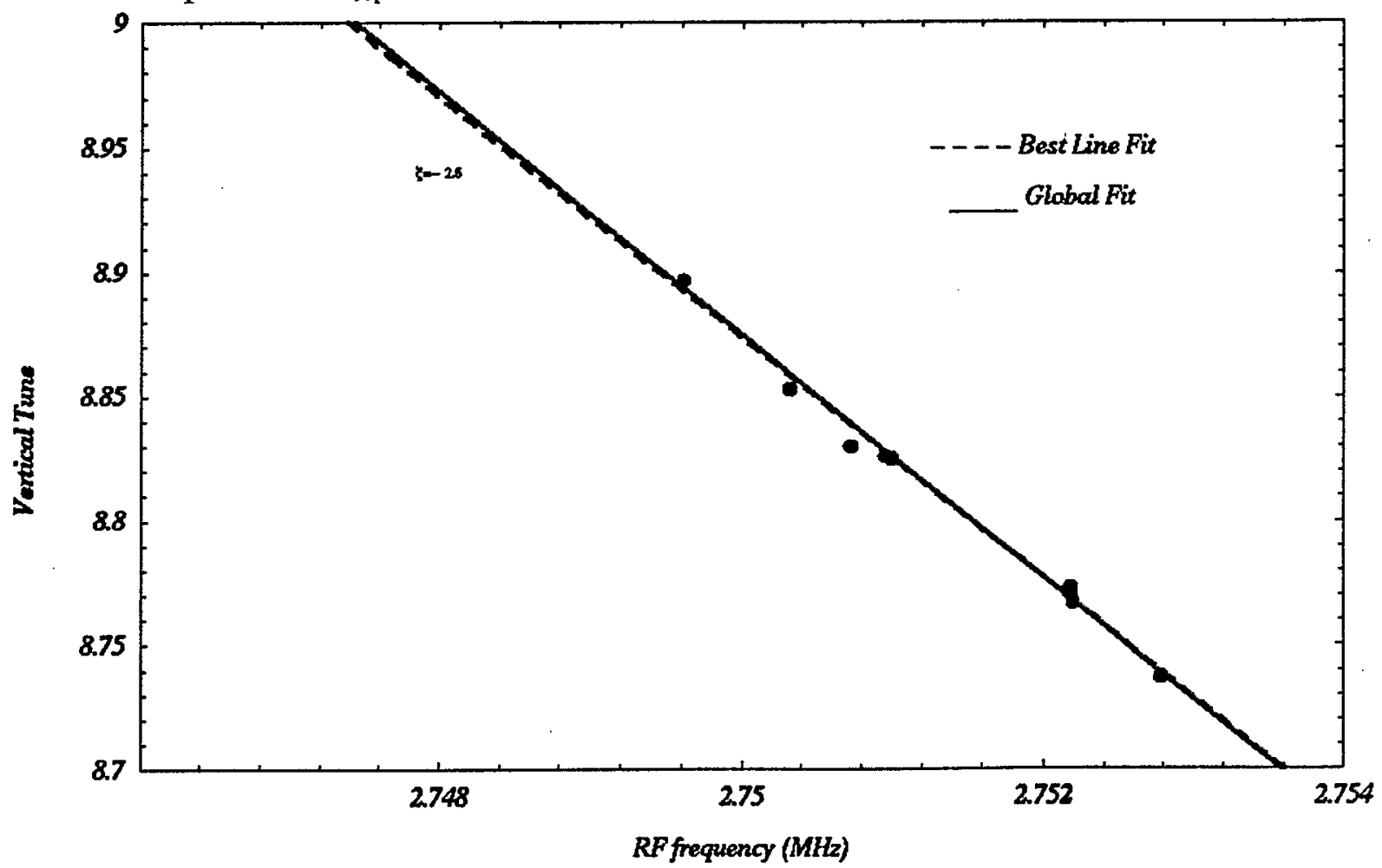

Figure 12 : Individual plot for best-fitted and global fitted equations for horizontal tune and $\xi=-2.6$. The dispersion between global fit and best-fitted equations corresponds to $\Delta \chi_{\mathrm{f}}^{2}=1.9$ 


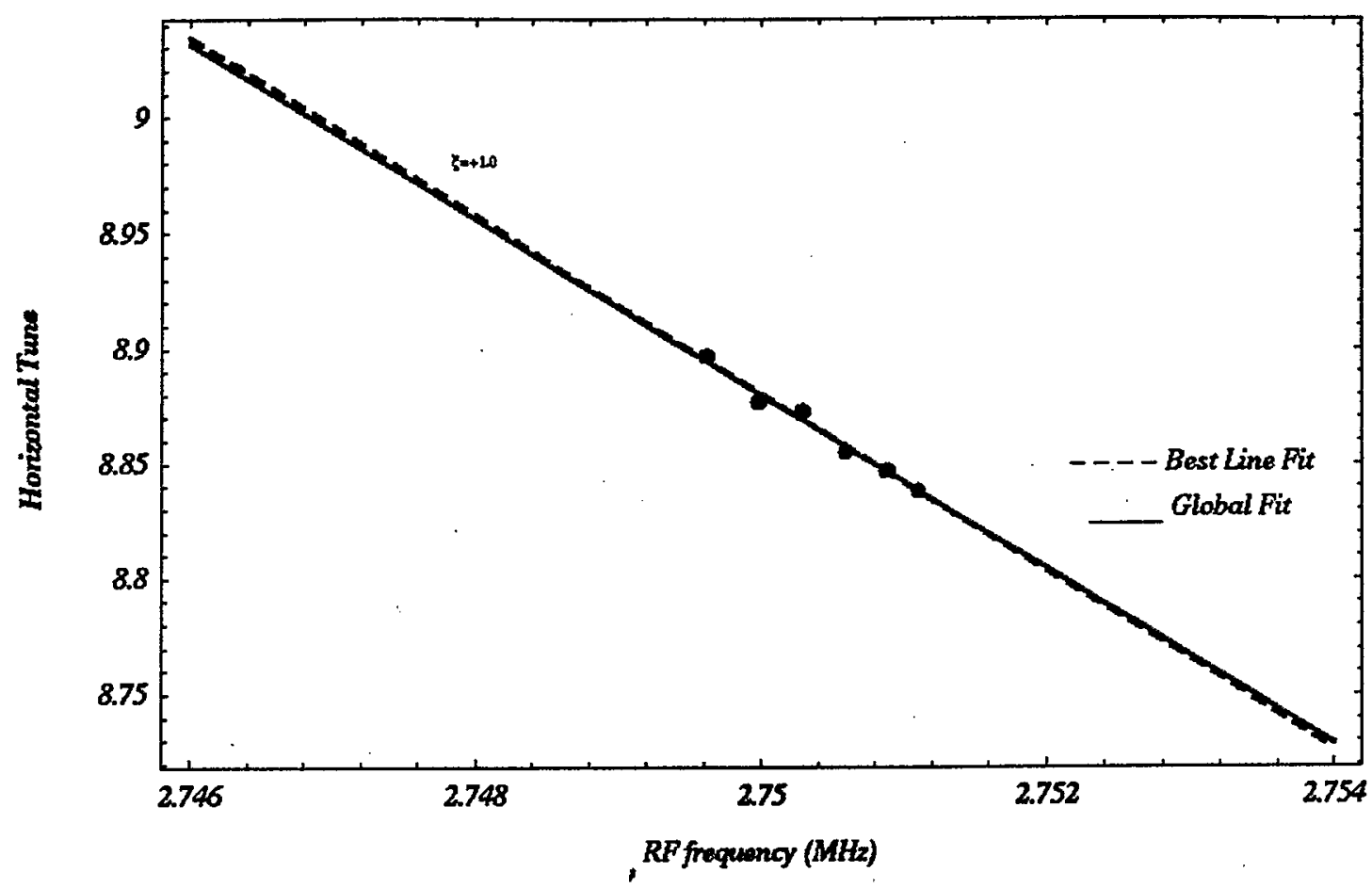

Figure 13 : Individual plot for best-fitted and global fitted equations for vertical tune and $\xi=+1.0$. The dispersion between global fit and best-fitted equations corresponds to $\Delta \chi_{\mathrm{f}}^{2}=0.7$

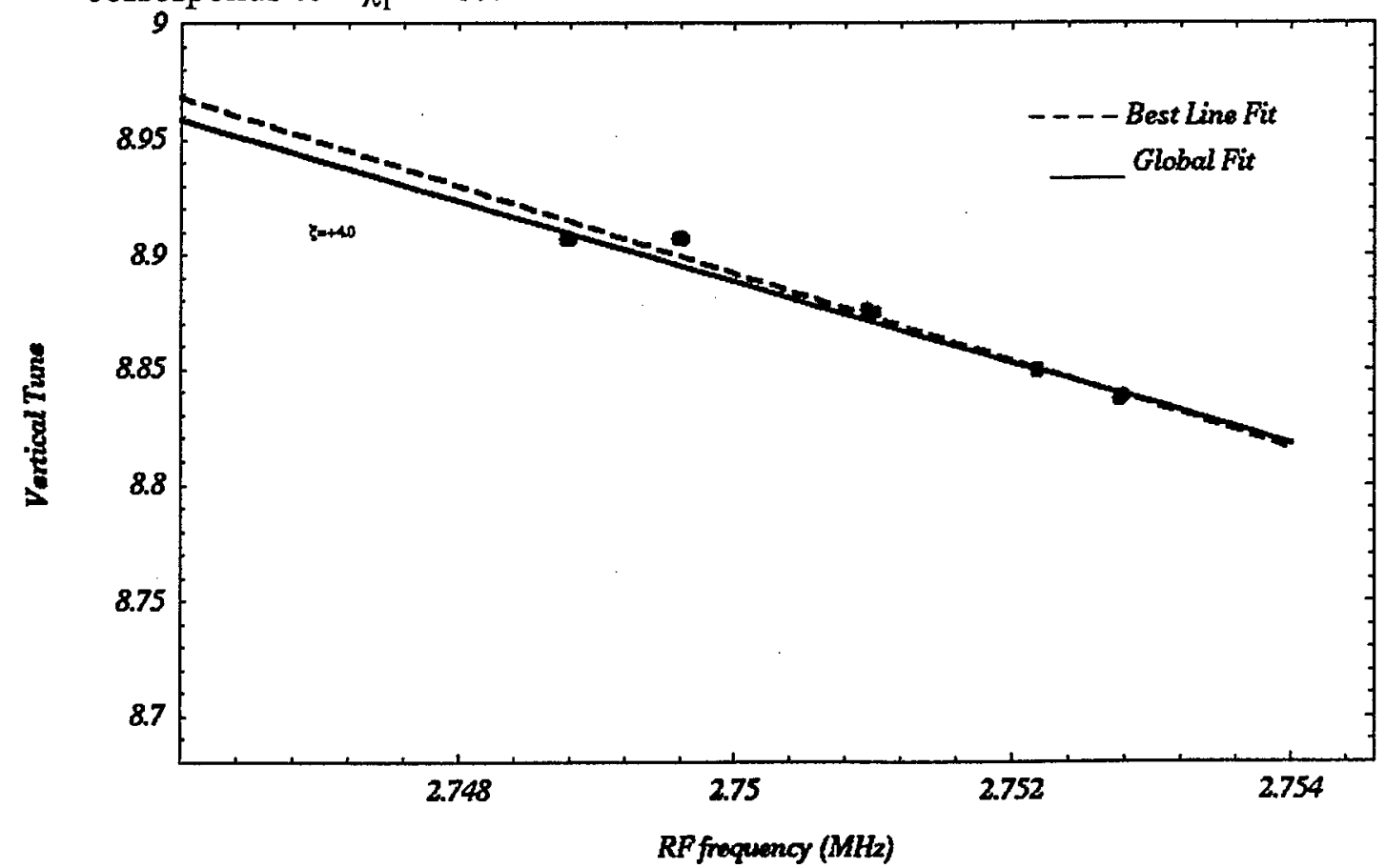

Figure 14 : Individual plot for best-fitted and global fitted equations for horizontal tune and $\xi=-4.0$. The dispersion between global fit and best-fitted equations corresponds to $\Delta \chi_{\mathrm{f}}^{2}=80.4$ 


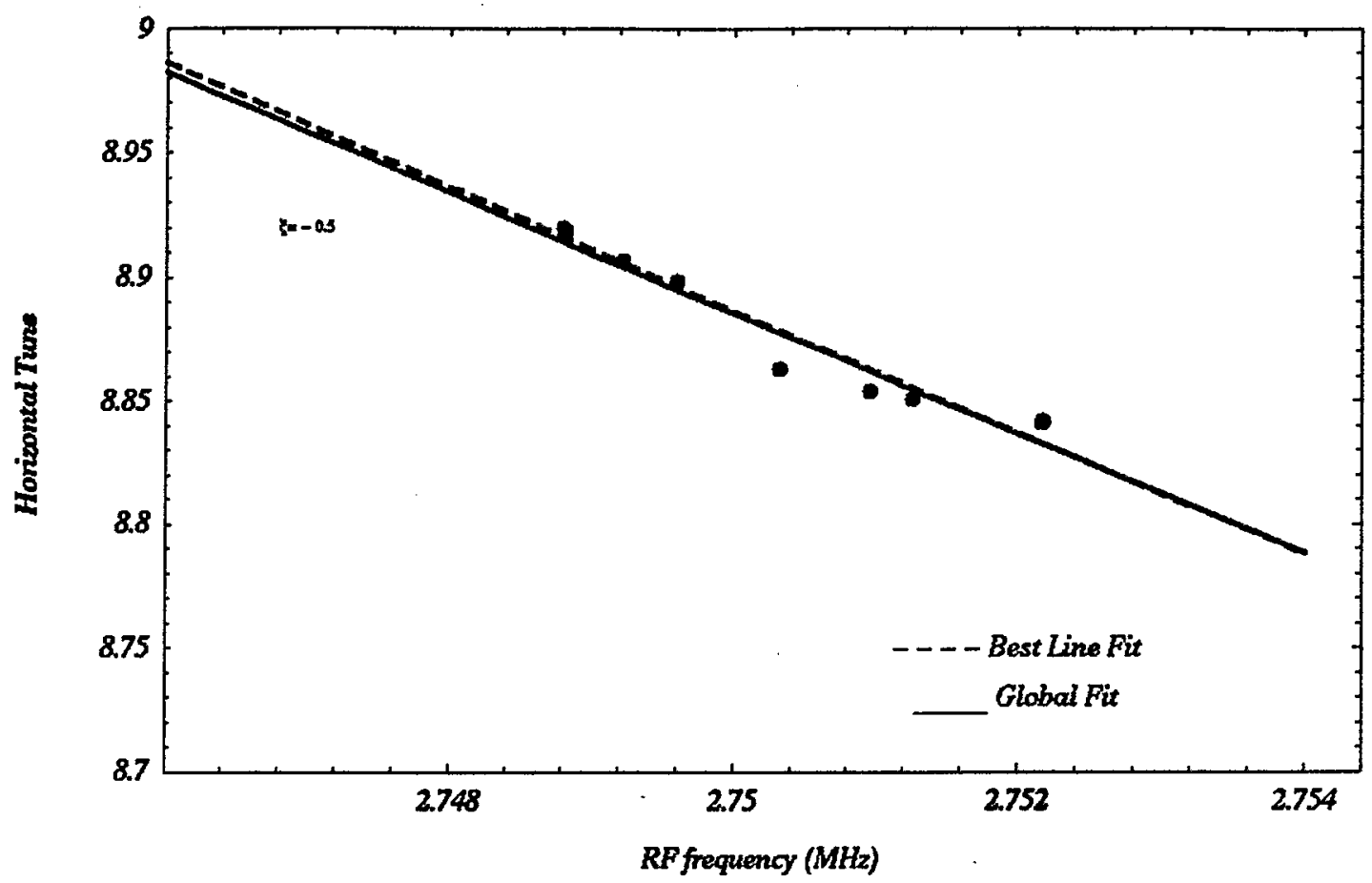

Figure 15 : Individual plot for best-fitted and global fitted equations for horizontal tune and $\xi=-0.5$. The dispersion between global fit and best-fitted equations corresponds to $\Delta \chi_{\mathrm{f}}^{2}=13.0$

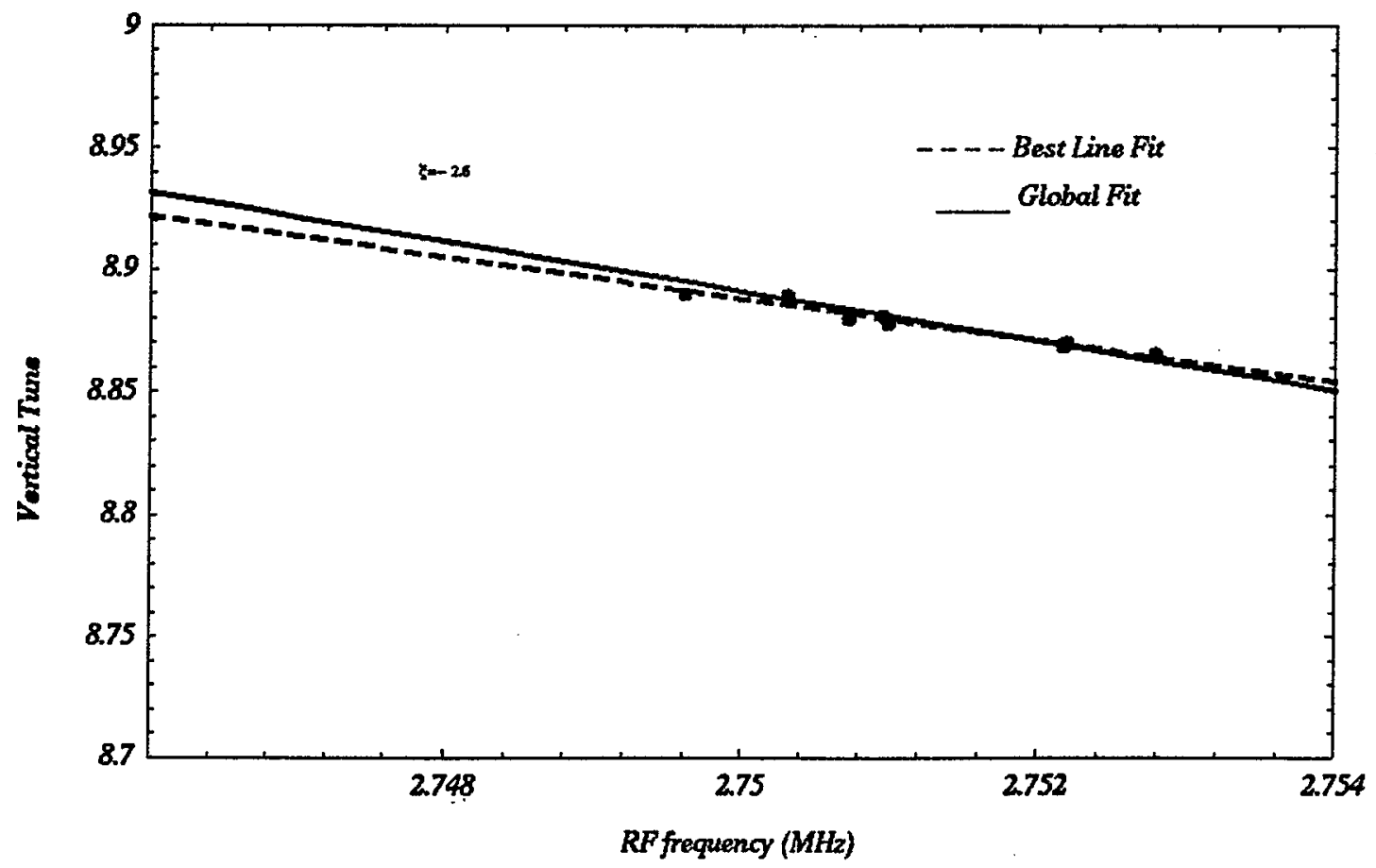

Figure 16 : Individual plot for best-fitted and global fitted equations for horizontal tune and $\xi=-2.6$. The dispersion between global fit and best-fitted equations corresponds to $\Delta \chi_{\mathrm{f}}^{2}=28.8$ 